\title{
A Complete End-To-End Open Source Toolchain for the Versatile Video Coding (VVC) Standard
}

\author{
Adam Wieckowski , Christian Lehmann ", Benjamin Bross , Detlev Marpe ", \\ Thibaud Biatek ${ }^{+}$, Mickael Raulet ${ }^{+}$, Jean Le Feuvre ${ }^{\$}$ \\ "Video Communication and Applications Department, Fraunhofer HHI, Berlin, Germany \\ ${ }^{+}$ATEME, Vélizy-Villacoublay, France \\ ${ }_{\text {LTCI, Telecom Paris, Institut Polytechnique de Paris, France }}$ \\ \{firstname.lastname\}@hhi.fraunhofer.de, \{t.biatek,m.raulet\}@ateme.com, jean.lefeuvre@telecom-paris.fr
}

\begin{abstract}
Versatile Video Coding (VVC) is the most recent international video coding standard jointly developed by ITU-T and ISO/IEC, which has been finalized in July 2020. VVC allows for significant bit-rate reductions around $50 \%$ for the same subjective video quality compared to its predecessor, High Efficiency Video Coding (HEVC). One year after finalization, VVC support in devices and chipsets is still under development, which is aligned with the typical development cycles of new video coding standards. This paper presents open-source software packages that allow building a complete VVC end-to-end toolchain already one year after its finalization. This includes the Fraunhofer HHI VVenC library for fast and efficient VVC encoding as well as HHI's VVdeC library for live decoding. An experimental integration of VVC in the GPAC software tools and FFmpeg media framework allows packaging VVC bitstreams, e.g. encoded with VVenC, in MP4 file format and using DASH for content creation and streaming. The integration of VVdeC allows playback on the receiver. Given these packages, step-by-step tutorials are provided for two possible application scenarios: VVC file encoding plus playback and adaptive streaming with DASH.
\end{abstract}

\section{CCS CONCEPTS}

- Computing methodologies Computer graphics Image compression $\bullet$ Software and its engineering $\sim$ Software creation and management $\sim$ Collaboration in software development $\sim$ Open source model

\section{KEYWORDS}

DASH; FFmpeg; GPAC; mp4; Open Source; Video Streaming; Versatile Video Coding (VVC); VVdeC; VVenC; HLS

\section{ACM Reference format:}

Adam Wieckowski, Christian Lehmann, Benjamin Bross, Detlev Marpe, Thibaud Biatek, Mickael Raulet, Jean Le Feuvre. 2021. A Complete End-ToEnd Open Source Toolchain for the Versatile Video Coding (VVC) Standard.

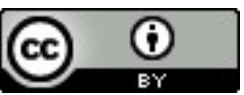

This work is licensed under a Creative Commons Attribution International 4.0 License.

MM '21, October 20-24, 2021, Virtual Event, China.

(c) 2021 Copyright held by the owner/author(s).

ACM ISBN 978-1-4503-8651-7/21/10.

https://doi.org/10.1145/3474085.3478320
In Proceedings of 29th ACM International Conference on Multimedia (MM'21), October 20-24, 2021, Virtual Event, China. ACM, New York, NY, USA, 4 pages. https://doi.org/10.1145/3474085.3478320

\section{Introduction}

In July 2020, the Versatile Video Coding (VVC) standard has been finalized by the Joint Video Experts Team (JVET) of ITU-T VCEG and ISO/IEC MPEG [1,2]. VVC was designed with two main objectives: significant bit-rate reduction over its predecessor High Efficiency Video Coding (HEVC) for the same perceived video quality and versatility to facilitate coding and transport for a wide range of applications and content types. This ranges from adaptive streaming of screen content to 360-degree video for virtual reality. The coding efficiency improvement of VVC over HEVC has been verified by independent subjective testing with naive viewers showing over $40 \%$ bit-rate reduction for High-Definition (HD) and Ultra-HD (UHD) video [3, 4] as well as over $50 \%$ bit-rate reduction for 360-degree video [4].

For standard development and verification, the VVC test model (VTM) reference software has been used. It is well suited to evaluate the compression potential of VVC but not optimized for runtime and lacks real-world features on the encoder side such as rate control, subjective optimizations, parallelization as well different efficiency/runtime trade-offs. Furthermore, VVC specifies the bitstream of coded video as well as the decoding processes. To use a video coding standard in real world application scenarios, its integration into transport systems layer such as MPEG-2 transport stream (MPEG2-TS) [5], ISO base media file format (ISOBMFF a.k.a. MP4 file format) [6] tracks is key. In addition, VVC encapsulated in MP4 file format can be used in dynamic adaptive streaming over HTTP (DASH) [7].

Real-world codec implementations in devices together with mp4 file format support for file-based playback and streaming typically emerge 2-3 years after the finalization of a standard. However, already today open-source software allows to use VVC with Fraunhofer HHI's encoder VVenC and decoder VVdeC. VVC bitstreams can be encapsulated in mp4 file format and packetized into DASH segments using GPAC while extraction and playback is feasible with VVdeC integrated into FFmpeg and GPAC. This paper describes these software packages in Section 2. Section 3 demonstrates how to use them for file playback and DASH 
streaming applications. Finally, Section 4 concludes this paper and gives an outlook of future work.

\section{VVC Toolchain Software Packages}

\subsection{VVenC}

The Fraunhofer Versatile Video Encoder, VVenC [8], is an optimized software encoder implementation of the VVC standard. Its source code is freely available on GitHub under a 3-clause BSD copyright license for both commercial and non-commercial use [9]. Version 1.0.0 has been released in May 2021, for which the results are presented.

VVenC has initially been derived from the VTM reference software. Its main purpose is to maintain the high coding efficiency of VTM while running significantly faster and providing real-world application features. While VVenC itself is implemented in $\mathrm{C}++$, the software package includes a $\mathrm{C}$ library interface as well as two sample applications that can be used as standalone command line encoders - a simple application designed for ease-of-use as well as an expert app resembling the VTM interface. VTM as well as VVenC convert a raw YUV video input into a VVC compliant bitstream.

2.1.1. Main features. Compared to the VTM software the encoder is much more user friendly. This includes five preconfigured presets allowing different encoding speed to compression efficiency trade-offs: faster, fast, medium, slow and slower. While the slower preset delivers full VTM compression performance at less than half of its runtime, other presets provide faster and much faster encoding at the cost of bitrate increase. Additional speedup can be obtained using multi-threading with minimal compression efficiency degradation. A combination of coding tree unit line and frame parallel processing provides very good thread number to runtime reduction scaling [10]. Single-pass

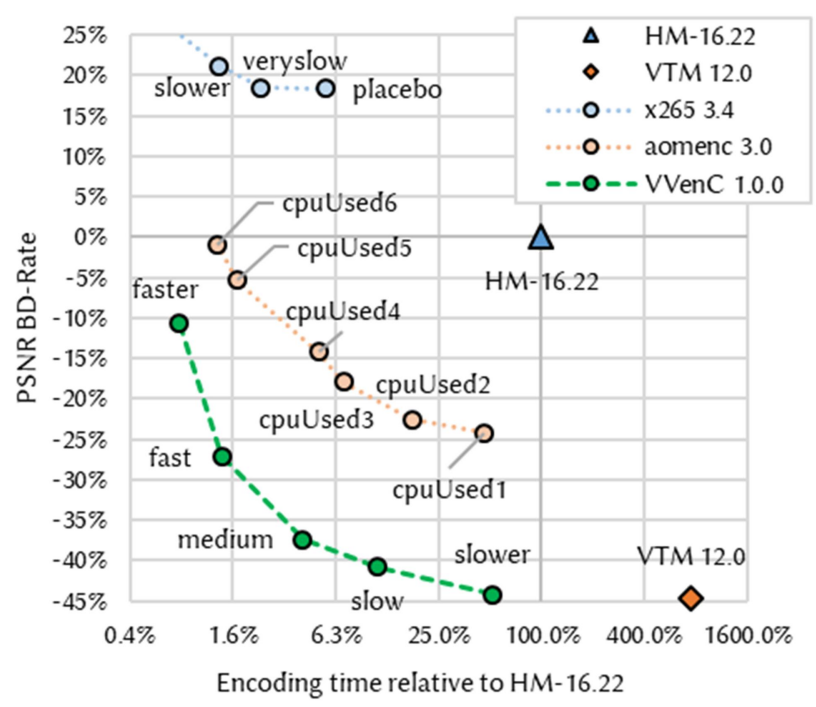

Figure 1: Runtime and BD rate of VVenC, $x 265$, aomenc and VTM compared to HM. All encoders but VTM and HM are run multithreaded with 8 threads. and two-pass frame-based rate control modes have been implemented, allowing very efficient encoding at fixed bit rates. Both have also been optimized for multi-threaded operation. VVenC further incorporates subjective optimizations using a weighted extended PSNR distortion metric (XPSNR). It overcomes the limited correlation of commonly used sum-of-squares- (SSD) or sum-of-absolutes (SAD) distortion calculations with perceived distortions using a simplified model of the human visual system to calculate weights. XPSNR can be calculated block-wise and therefore incorporated into block-level rate-distortion decisions. It is implemented by the means of local quantization parameter adaptations (QPA) and designed to improve the rate control accuracy and performance as well [11]. As of the current version, VVenC is compliant to the Main 10 profile (4:2:0 chroma subsampling and up to 10 bit per sample) and optimized for standard dynamic range (SDR) as well as for high dynamic range (HDR) content with wide color gamut. Currently, only one slice and tile per picture is supported, without sub-pictures. VVenC already anticipates versatile application scenarios by incorporating all Main 10 profile screen content coding tools and providing a constrained encoding mode optimized for use in open-GOP adaptive streaming with resolution change [12].

2.1.2. Results. The multi-threaded runtime and PSNR-based Bjøntegard Delta (BD) rate compression performance (negative numbers mean bit-rate reduction) for VVenC 1.0.0 relative to the HEVC reference software (HM-16.22) is shown in Figure 1. The results are based on encoding of the HD and UHD sequences from the JVET common test conditions [13] test-set under JVET random access conditions. Additionally, the figure contains results for VTM 12.0 as well as two alternative freely available encoders, for HEVC (x265 3.4) and AV1 (aomenc 3.0). The results show that VVenC can achieve VTM coding efficiency at much lower runtimes when run multi-threaded. Compared to aomenc, it provides a steady $20 \%$ bit-rate reduction at similar runtimes. The three faster presets of VVenC can match the three slowest x265 presets regarding encoding time with very high bit-rate savings of around $50 \%$. However, there are no VVenC presets available yet to match the encoding speed in faster $\mathrm{x} 265$ presets.

In recent JVET VVC verification tests, initial versions of VVenC were subjectively tested together with VTM and HM for UHD (VVenC 0.1) and HD (VVenC 0.3). These tests, which have been conducted in a controlled laboratory environment with naive viewers, concluded that VVenC in medium preset with subjective optimizations visually outperformed VTM.

\subsection{VVdeC}

The Fraunhofer Versatile Video Decoder, VVdeC, is an optimized VVC software decoder implementation, freely available on GitHub under a 3-clause BSD copyright license [14]. Analogue to VVenC, the license covers both commercial and non-commercial use. The latest release, v1.1.2, was published in July 2021. It is compliant with the VVC Main 10 profile and correctly decodes the current VVC conformance testing set [15].

The decoder software has been derived from VTM as well, with subsequent optimizations and parallelization. Being based on VTM, it has performance limitations beyond the complexity of the 
VVC standard itself. The decoder allows playback of HD video at 60 frames per second (fps) on most modern computers using only 2 or 3 threads, and 60 fps UHD playback on more powerful modern workstations with sufficient processing cores to fully exploit the multi-threading potential [16].

The VVdeC package contains a simple and easy-to-use C library interface and a standalone decoder application capable of decoding elementary VVC bitstreams into raw YUV video data. The framework integrations described in the following sections allow actual video playback.

\subsection{FFmpeg VVC integration with VVdeC}

FFmpeg is a well-known multimedia framework which provides a set of libraries to record, convert and stream any type of audio and video data. In order to support VVC in FFmpeg, a parser and a Code Bitstreams Type (CBT) were implemented in the development branch (currently under review). This first step enables support for VVC elementary streams but does not provide a system level support with standard delivery mechanisms such as MPEG2-TS or DASH. To further extend the VVC capabilities in FFmpeg, the support for VVdeC and MPEG2-TS as well as ISOBMFF/DASH binds have been implemented by ATEME in a public FFmpeg fork [17]. The support for VVC video stream type has been added to MPEG2-TS demuxer in the libavformat. The ISOBMFF demuxer has been upgraded to support VVC tag 'vvc1' and 'vvcC' MOV table entry. Finally, the VVC MP4-to-AnnexB filter has been implemented in libavcodec for proper demuxing and decoding.

The provided implementation enables FFmpeg to natively support VVC services when delivered in MPEG2-TS or DASH. In addition, it provides VVC support through libavcodec when integrated in other projects, such as GPAC.

Within this fork, ffplay can be used to playback DASH manifest, or UDP MPEG2-TS streams, by using:

ffplay -i URL/manifest.mpd

ffplay -f mpegts -i udp://host:port

\subsection{VVC support in GPAC}

The GPAC multimedia framework provides a set of tools to package, stream and playback multimedia content. It is wellknown for its MP4Box tool, an mp4 file packager and HTTP streaming (HLS) segmenter. GPAC also enables end users to build custom multimedia processing pipelines through its filter-based architecture [18].

The systems aspect of VVC have been implemented in the master branch of GPAC and are part of the nightly builds of the project. This covers the most common application use cases:

- MPEG-2 broadcasting: multiplexing and demultiplexing an MPEG2-TS program with VVC content

- $\quad$ MP4 file packaging and dumping of VVC bitstreams

- $\quad$ MPEG-DASH and HLS content packaging

GPAC supports all codecs integrated in FFmpeg's libavcodec library, including VVdeC as included in [17]. This is detected at configure time and currently requires a custom build of GPAC, VVdeC and libavcodec, as previously explained. The GPAC player embeds the customized libavcodec which itself embeds the VVdeC core decoding library.

Until integration of VVenC is finalized in libavcodec, GPAC can consume the VVenC output through files and pipes.

\section{Application scenarios}

In this section, we give some examples of using VVC in common case scenarios. The described end-to-end chain addresses both MPEG2-TS and IP based delivery mechanisms.

When used in a traditional digital video broadcast terrestrial/satellite environment (DVB-T2/S2), VVC bitstreams encapsulated in MPEG2-TS can be efficiently delivered and played with the proposed framework. The FFmpeg player has been used for that purpose during an in-field VVC broadcasting trial [19] showing interoperability with legacy delivery infrastructure. Recent full-IP broadcasting technologies such as ATSC-3.0 or the currently under-standardization TV3.0 in Brazil have also been addressed. The VVC encapsulation and carriage with ISOBMFF opens the door for such modern applications where the proposed solution can be used in an end-to-end manner. VVenC can be used to prepare and encode the video sequence (Figure 2), while GPAC can package and multicast the services using DASH / ROUTE, in an ATSC-3.0 fashion. At the receiver side, the GPAC client integrated with libavcodec/VVdeC can be used to deliver TV services to the viewer (Figure 2). The proposed solution is currently part of a response to the SBTVD TV3.0 [20] call for technology, utilizing presented components for playback.

In the following subsections two encoding and distribution scenarios using VVC are demonstrated step-by-step.

\subsection{VVC encoding and packaging for playback}

VVenC requires a YUV input file, either existing or decoded from another video file. Starting from the raw source video file, the

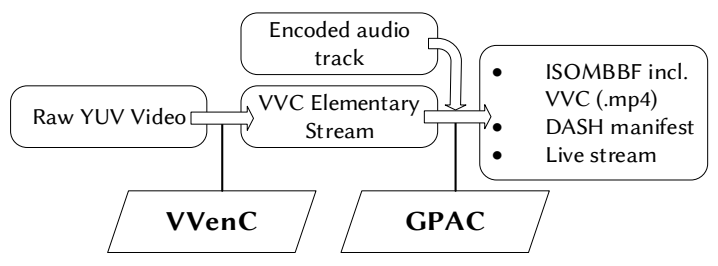

a) Encoding workflow and used components.

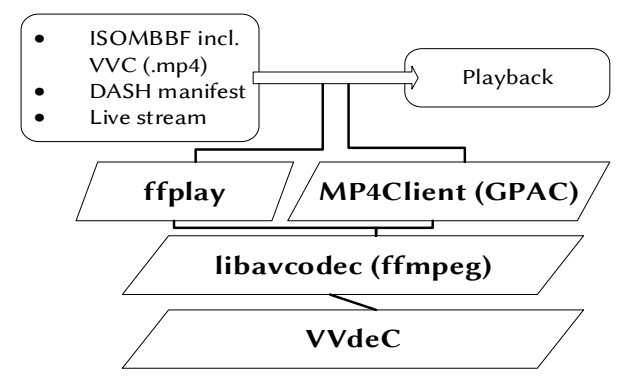

b) Decoding workflow and components.

Figure 2: Components used in en- and decoding. 
following steps are required to create a packaged VVC encode of the raw YUV input input.yuv:

- (Optional) decode an existing video to 8-bit YUV input.yuv

gpac -i input.mp4 -o input.yuv

- $\quad$ Encode the raw YUV data to VVC elementary stream es.vvc

vvencapp --preset medium -i input.yuv -s

1920×1080 [OPTS] -o es.Vvc

- Possible packaging options with GPAC

- Package the ES as MP4 to VVC_demo.mp4

MP4Box -add es.vvc -new VVC_demo.mp4

- Mux raw VVC and AAC streams to MP4

MP4Box -add es.vvc -add audio.aac -new

VVC_demo.mp4

- Create an MPEG-2 TS live-stream at 10mbps (VVenC bitstreams might exceed some rate constraints)

gpac -i es.vvc -i audio.aac -o udp://127.

$0.0 .1: 1234 /:$ ext=ts: rate $=10 \mathrm{~m}:$ realtime

In the presented examples the VVC elementary stream (ES) file extension does not matter, because GPAC including MP4Box always probe the input data.

The following commands enable MP4 playback and high-level inspection of a VVC bitstream:

MP4Client -gui URL

gpac -i URL inspect: deep: anayze=bs

\subsection{VVC encoding and packaging for DASH}

HTTP streaming works by segmenting the input media into short segments, each starting with an IDR frame. Some configurations will expect a roughly constant segment duration, which may require enforcing fixed frame structure. VVenC can be forced to create appropriate encoding for DASH using the parameters --refreshtype idr.

GPAC accepts both raw bitstreams or packaged files, such as MP4, as input to its DASH preparation process. The creation of DASH sessions in different profiles can be acchieved using:

- Using the "onDemand" profile:

MP4Box -dash 2000 -profile onDemand es.vvc -out dash/vod.mpd

- Using the "live" profile:

MP4Box -dash 2000 -profile live es.vvc -out dash/vod.mpd:dual

- Using segment timeline for variable duration segments: MP4Box -dash 2000 -profile live es.vvc -out dash/vod.mpd:st I

The following illustrates creating live DASH and HLS sessions made available through GPAC HTTP server:

gpac flist:srcs $=V V C$ AAC $\cdot m p 4: f l o p=-1$ reframer $: r t$ =on \& -o http://127.0.0.1:8080/live.mpd:gpac: dual $:$ segdur $=2:$ cdur $=0.1:$ asto $=1.9: 1 \mathrm{lhls}=\mathrm{br}:$ dmode $=\mathrm{d}$ ynamic:rdirs=dash_live

More information regarding the complex subject of HTTP Streaming is available on GPAC's wiki, including HowTos.

\section{Conclusion and Future Work}

This paper teaches how to use publicly available open-source software to set up a complete VVC toolchain for file playback and adaptive streaming application scenarios.

Beyond these more traditional use cases, future work will focus on novel applications like adaptive streaming utilizing resolution change or high-resolution streaming with tiles for VR applications.

\section{REFERENCES}

[1] ITU-T and ISO/IEC JTC 1. 2020. Versatile Video Coding, Rec. ITU-T H.266 and ISO/IEC 23090-3 (VVC)

[2] B. Bross, J. Chen, J.-R. Ohm, G. J. Sullivan and Y.-K. Wang. 2021. Developments in International Video Coding Standardization After AVC, with an Overview of Versatile Video Coding (VVC). Proc. of the IEEE (fan. 2021), 31 pages. DOI: https://doi.org/10.1109/JPROC.2020.3043399

[3] V. Baroncini and M. Wien. 2020. VVC verification test report for UHD SDR video content. Doc. JVET-T2020 of ITU-T/ISO/IEC Joint Video Experts Team (JVET), 20th JVET meeting: October 2020.

[4] V. Baroncini and M. Wien. 2021. Dry run subjective assessment of SDR HD and 360 video verification tests. Doc. JVET-U0119 of ITU-T/ISO/IEC Joint Video Experts Team (JVET), 21st JVET meeting: January 2021.

[5] K. Grüneberg, Y. Lim, Y. Syed, and P. Wu (eds.). 2020. Text of ISO/IEC 138181:2019 DAM 2 Carriage of VVC in MPEG-2 TS. ISO/IEC JTC 1 SC 29 WG 11 output document N19436.

[6] K. Grüneberg, M. M. Hannuksela, J. M. Le Fevre, and Y.-K. Wang (eds.). 2020. Potential improvements on Carriage of VVC and EVC in ISOBMFF, ISO/IEC JTC 1/SC 29 WG 03 output document N0035.

[7] ISO/IEC JTC 1. 2012. Information technology-Dynamic adaptive streaming over HTTP (DASH) - Part 1: Media presentation description and segment formats, ISO/IEC 23009-1.

[8] A. Wieckowski, J. Brandenburg, T Hinz, C. Bartnik, V. George, G. Hege, C. Helmrich, A. Henkel, C. Lehmann, C. Stoffers, I .Zupancic, B. Bross and D. Marpe. 2021. VVenC: An Open And Optimized VVC Encoder Implementation. In 2021 IEEE International Conference on Multimedia \& Expo Workshops (ICMEW) July $5-9, \quad 2021, \quad$ Shenzhen, China. $1-2 . \quad$ DOI: https://doi.org/10.1109/ICMEW53276.2021.9455944

[9] Fraunhofer HHI VVenC software repository. Retrieved from https://github.com/fraunhoferhhi/vvenc.

[10] VVenC Fraunhofer Versatile Video Encoder v1.0.0. Retrieved from https://github.com/fraunhoferhhi/vvenc/wiki/data/vvenc-v1.0.0-v1.pdf.

[11] C. R. Helmrich, I. Zupancic, J. Brandenburg, V. George, A. Wieckowski and B. Bross. 2021. Visually Optimized Two-Pass Rate Control for Video Coding Using the Low-Complexity XPSNR Model. Submitted to VCIP'21.

[12] R. Skupin, C. Bartnik, A. Wieckowski, Y. Sanchez, B. Bross, C. Hellge, and T. Schierl. 2021. Open GOP Resolution Switching in HTTP Adaptive Streaming with VVC. In 2021 Picture Coding Symposium (PCS), June 29 - July 2, 2021, Bristol, UK. DOI: https://doi.org/10.1109/PCS50896.2021.9477501

[13] F. Bossen, J. Boyce, X. Li, V. Seregin, and K. Sühring. 2020. FVET common test conditions and software reference configurations for SDR video. Doc. JVET-T2010 of ITU-T/ISO/IEC Joint Video Experts Team (JVET), 20th JVET meeting: October 2020.

[14] Fraunhofer HHI VVdeC software repository. Retrieved from https://github.com/fraunhoferhhi/vvdec.

[15] J. Boyce, E. Alshina, F. Bossen, K. Kawamura, I. Moccagatta and W. Wan. 2021 Conformance testing for versatile video coding (Draft 6). Doc. JVET-U2008 of ITUT/ISO/IEC Joint Video Experts Team (JVET), 21st JVET meeting: January 2021.

[16] A. Wieckowski, G. Hege, C. Bartnik, C. Lehmann, C. Stoffers, B. Bros, and D. Marpe. 2020. Towards a Live Software Decoder Implementation for the Upcoming Versatile Video Coding (VVC) Codec. In 2020 IEEE International Conference on Image Processing (ICIP), October, 2020, Abu Dhabi, UAE 3124-3128. DOI: https://doi.org/10.1109/ICIP40778.2020.9191199

[17] FFmpeg fork with full VVdeC integration. Retrieved from https://github.com/tbiat/FFmpeg/releases/tag/vvc.

[18] Jean Le Feuvre. 2020. GPAC filters. In Proceedings of the 11th ACM Multimedia Systems Conference. ACM, New York, NY, USA, 249-254. DOI: https://doi.org/10.1145/3339825.3394929

[19] T. Biatek, M. Abdoli, T. Guionnet, M. Raulet, T. Wrede, J. Outters, T. Christophory, H. Bauzée-Luyssen, S. Latapie, J.-B. Kempf, P.-L. Cabarat, and W. Hamidouche. 2020. End-to-End UHD Satellite Broadcast Transmission using VVC. MPEG document m54377.

[20] TV3.0 Project. Retrieved from https://forumsbtvd.org.br/tv3_0/ 